\title{
Clinical Efficacy of Perioperative Immunonutrition Containing Omega-3-Fatty Acids in Patients Undergoing Hepatectomy: A Systematic Review and Meta-Analysis of Randomized Controlled Trials
}

\author{
Benjian Gao ${ }^{a}$ Jia Luo ${ }^{b}$ Ying Liu ${ }^{a}$ Furui Zhonga ${ }^{a}$ Xiaoli Yanga Yu Gan ${ }^{a}$ \\ Song Su${ }^{a}$ Bo $\mathrm{Li}^{\mathrm{a}}$ \\ aDepartment of Hepatobiliary Surgery, The Affiliated Hospital of Southwest Medical University, Luzhou, China; \\ ${ }^{b}$ Department of Oncology, The Affiliated Hospital of Southwest Medical University, Luzhou, China
}

\section{Keywords}

Immunonutrition · Hepatectomy · Randomized controlled trials $\cdot$ Meta-analysis

\begin{abstract}
Background: The effect of immunonutrition in patients undergoing hepatectomy remains unclear. This meta-analysis aimed to assess the impact of immunonutrition on postoperative clinical outcomes in patients undergoing hepatectomy. Methods: A literature search of PubMed, Cochrane Library, Web of Science, and Embase databases was performed to identify all randomized controlled trials (RCTs) exploring the effect of perioperative immunonutrition in patients undergoing hepatectomy until the end of March 10, 2020. Quality assessment and data extraction of RCTs were conducted independently by 3 reviewers. Mean difference (MD) and odds ratio (OR) with $95 \%$ confidence interval $(\mathrm{Cl})$ were calculated using a fixed-effects or random-effects model. The meta-analysis was performed with RevMan 5.3 software. Results: Nine RCTs involving a total of 966 patients were finally included. This meta-analysis showed that immunonutrition significantly reduced the incidences of overall postoperative complications $(\mathrm{OR}=0.57,95 \% \mathrm{Cl}$ : 0.34-0.95; $p=$
\end{abstract}

karger@karger.com

(C) 2020 S. Karger AG, Basel

www.karger.com/anm

Karger"
$0.03)$, overall postoperative infectious complications ( $\mathrm{OR}=$ 0.53 , 95\% Cl: $0.37-0.75 ; p=0.0003$ ), and incision infection $(\mathrm{OR}=0.50,95 \% \mathrm{Cl}: 0.28-0.89 ; p=0.02)$, and it shortened the length of hospital stay ( $\mathrm{MD}=-3.80,95 \% \mathrm{Cl}:-6.59$ to -1.02 ; $p=0.007)$. There were no significant differences in the incidences of pulmonary infection $(\mathrm{OR}=0.60,95 \% \mathrm{Cl}: 0.32-1.12$; $p=0.11)$, urinary tract infection $(\mathrm{OR}=1.30,95 \% \mathrm{Cl}: 0.55-3.08$; $p=0.55)$, liver failure $(\mathrm{OR}=0.54,95 \% \mathrm{Cl}: 0.23-1.24 ; p=0.15)$, and postoperative mortality (OR $=0.69,95 \% \mathrm{Cl}: 0.26-1.83$; $p=0.46)$. Conclusion: Given its positive impact on postoperative complications and the tendency to shorten the length of hospital stay, perioperative immunonutrition should be encouraged in patients undergoing hepatectomy.

(c) 2020 S. Karger AG, Basel

\section{Introduction}

Hepatectomy is the mainstay curative treatment for benign and malignant hepatobiliary diseases [1]. Despite advances in surgical technique and perioperative management in recent decades, the postoperative mortality and

Jia Luo and Benjian Gao contributed equally to this work. 
morbidity rates associated with hepatectomy remain high; they range from 1.0 to $5.0 \%$ and 15.6 to $40.0 \%$, respectively [2-6]. In patients suffering from chronic or malignant liver diseases, malnutrition is prevalent and considered to be a predictive factor for increased morbidity and mortality rates after surgery [7-9]. From a nutritional point of view, perioperative nutritional management and optimization may improve the hepatic function and reduce the postoperative complications in patients treated with liver resection [10]. Recently, the main focus of nutritional management has not been solely to provide energy and nitrogen, but to supplement selected nutritional substrates, which have specific pharmacological effects.

The immunonutrition formula, which generally includes nutrients such as $\omega$-3-fatty acids ( $\omega$-3-FAs), glutamine (Gln), arginine (Arg), and nucleotides, seems to be a promising treatment [11]. In addition to ensuring nitrogen balance and protein synthesis, the supply of immunonutrition can modulate the inflammatory response, counteract postoperative immune impairment, and improve clinical outcomes [12]. Some previous studies have indicated that perioperative immunonutrition support in patients undergoing elective gastrointestinal surgery could significantly modulate the inflammatory reaction, improve cellular immunity, and reduce postoperative infectious complications and length of hospital stay compared with that in patients fed standard nutrition [13-16]. Besides, both malnourished and well-nourished patients can benefit from immunonutrition [17]. Moreover, perioperative immunonutrition in patients undergoing major abdominal surgery has been recommended by the European Society for Parenteral and Enteral Nutrition (ESPEN) and implemented in the national guidelines $[18,19]$.

A recent meta-analysis of randomized controlled trials (RCTs) comparing immunonutrition versus standard nutrition in patients undergoing hepatectomy showed positive effects of immunonutrition on the liver function and inflammatory reaction [20]. However, this study failed to assess clinical outcomes, such as postoperative complications, mortality, and length of hospital stay. In addition, several studies [21-29] have been conducted to evaluate the effectiveness of immunonutrition support in patients undergoing liver resection, and they have yielded inconsistent results. Therefore, whether immunonutrition is beneficial and safe for these patients remains undetermined. Herein, this meta-analysis was conducted to systematically review the published RCTs and to evaluate the clinical efficacy of perioperative immunonutrition in patients undergoing hepatectomy, thus aiming to provide more reliable evidence for clinical decision.

\section{Methods}

This review was performed in accordance with the guidelines of the Preferred Reporting Items for Systematic Reviews and MetaAnalyses (PRISMA) Statement [30]. The prospective protocol for this study has been registered in the International Prospective Register of Systematic Review (PROSPERO), and the registration number is CRD42020163591.

\section{Search Strategy}

We systematically searched the PubMed, Cochrane Library, Web of Science, and Embase databases for all RCTs exploring the clinical efficacy of perioperative immunonutrition supplementation in patients undergoing liver resection. The following search terms were included: hepatectomy, hepatectomies, liver resection, hepatic resection, liver surgery, liver operation, immunonutrition, immune nutrients, immune nutrition, immuno-enhanced nutrition, omega-3-fatty acids, $\omega$-3-fatty acids, $\omega$-3-FAs, arginine, Arg, glutamine, Gln, nucleotides, RNA, and randomized controlled trials. The reference lists of the included studies were also reviewed to identify other potentially eligible studies. The literature search was limited to articles written in English language and dated through March 10, 2020.

\section{Inclusion and Exclusion Criteria}

Two authors (B.G. and J.L.) independently screened the results of initial searches. Studies that met the following criteria were included in our meta-analysis: (1) RCTs; (2) the experimental group received perioperative immunonutrition supplementation (including $\omega$-3-FAs or Gln or Arg or nucleotides) as the only intervention for patients who underwent hepatectomy, while the control group received placebo or regular nutrition; (3) studies reporting at least one of the following outcomes: postoperative complications, liver failure, postoperative mortality, and length of hospital stay; and (4) availability of full text. Exclusion criteria were as follows: (1) studies without a control group; (2) case reports, letters, reviews, conference reports, or experiments; and (3) conference abstracts without the full text.

\section{Data Extraction}

Three authors (B.G., J.L., and Y.L.) read the full text of the eligible studies and extracted data independently. Any discrepancies or disagreements were solved through discussion in plenum. Data extraction included the first author, title and date of publication, country, age, gender, sample size, types and timing of intervention, and route of nutrition. In this meta-analysis, outcomes of interest recorded included overall postoperative complications, postoperative infectious complications, incision infection, pulmonary infection, urinary tract infection, liver failure, postoperative mortality, and length of hospital stay.

\section{Quality Assessment}

The Cochrane Collaboration's tool for assessing risk of bias [31] was used to evaluate the quality of the selected studies, and it contained the following 7 items: random sequence generation, allocation concealment, blinding of participants and personnel, blinding of outcome assessment, incomplete outcome data, selective outcome reporting, and other bias. Risk of bias was graded as "low risk," "high risk," or "unclear risk." The results were shown as risk of bias graph and risk of bias summary. 


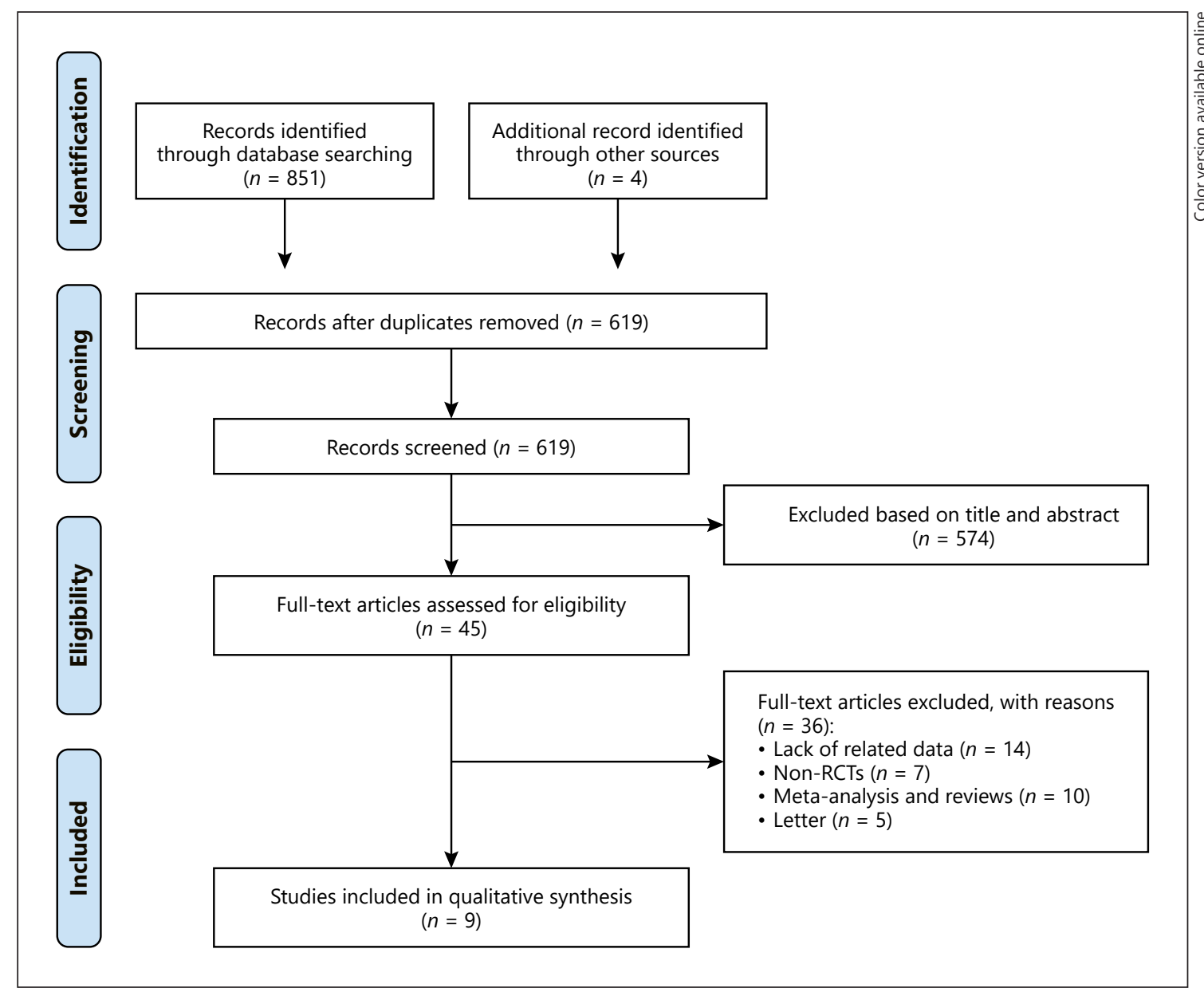

Fig. 1. Flow diagram of literature selection. RCTs, randomized controlled trials.

\section{Statistical Analysis}

Statistical analyses were conducted using the Cochrane Review Manager software (RevMan, version 5.3). Categorical variables were expressed as odds ratio (OR) with 95\% confidence interval (CI), and continuous variables were assessed by mean difference (MD) with 95\% CI. Between-study heterogeneity was evaluated using the $\chi^{2}$ test and $I^{2}$ statistics. Besides, heterogeneity was regarded as significant when the $p<0.1$ or $I^{2}>50 \%$. The randomeffects model was used if heterogeneity was significant; otherwise, the pooled analysis was carried out with the fixed-effects model. The funnel plot was not drawn to evaluate the potential publication bias due to the limited number $(<10)$ of articles included in each analysis. Sensitivity analysis was performed by removing each study in turn. A $p<0.05$ was considered statistically significant.

\section{Results}

\section{Literature Search and Study Characteristics}

A total of 855 articles were identified through the initial search, of which 236 studies were discarded because of duplication and 574 records were excluded after reading the titles and abstracts. Whole 45 studies were eligible for further full-text screening, and 36 studies were excluded because they did not meet the inclusion criteria: 7 were not RCTs, 10 were systematic reviews, 5 were letters, and 14 articles lacked the related data. Finally, 9 articles were included in the analysis [21-29]. A flow diagram of the search strategy is presented in Figure 1.

Table 1 details the characteristics of the included RCTs. All eligible studies included a total of 966 participants: 485 in the experiment group and 481 in the control group. Five $[21,22,24,26,27]$ of these 9 studies were from Asia, 3 studies $[23,25,28]$ were conducted in Europe, and 1 study [29] was performed in Oceania. The sample size of the study ranged from 25 [25] to 312 [27]. Four out of the 9 trials were performed to compare immunonutrition with regular nutrition [22, 24, 26, 27], 3 trials were performed for comparing immunonutrition with placebo 
Table 1. Characteristics of the included studies

\begin{tabular}{|c|c|c|c|c|c|c|c|}
\hline Author & Country & $\begin{array}{l}\text { Sample size } \\
\text { (Exp/Cont) }\end{array}$ & $\begin{array}{l}\text { Age, years } \\
\text { (Exp/Cont) }\end{array}$ & $\begin{array}{l}\text { Timing of } \\
\text { IN initiation }\end{array}$ & Nutrients of IN & $\begin{array}{l}\text { Route } \\
\text { of IN }\end{array}$ & $\begin{array}{l}\text { Duration } \\
\text { of IN, days }\end{array}$ \\
\hline Mikagi et al. [21] & Japan & $13 / 13$ & $67.5 \pm 11.3 / 61.5 \pm 10.2$ & Preoperative & IMPACT (Arg, $\omega-3-F A s$, and RNA) & EN & 5 \\
\hline Cockbain et al. [23] & UK & $43 / 45$ & $68(44-82) / 71(35-87)$ & Preoperative & $\omega$-3-FAs & EN & NA \\
\hline Gong et al. [24] & China & $59 / 60$ & $51.4 \pm 11.8 / 49.7 \pm 11.2$ & Postoperative & $\omega$-3-FAs & $\mathrm{PN}$ & 5 \\
\hline Seguin et al. [25] & France & $13 / 12$ & $65 \pm 8 / 68 \pm 6$ & Preoperative & IMPACT (Arg, $\omega-3-F A s$, and RNA) & $\mathrm{EN}$ & 7 \\
\hline Linecker et al. [28] & Switzerland & $132 / 129$ & $59(44-68) / 60(50-68)$ & Preoperative & $\omega$-3-FAs & $\mathrm{PN}$ & 2 \\
\hline Russell et al. [29] & New Zealand & $17 / 15$ & $61(28-76) / 63(31-79)$ & Preoperative & IMPACT (Arg, $\omega-3$-FAs, and RNA) & EN & 5 \\
\hline
\end{tabular}

NA, not available; Exp, experiment group; Cont, control group; IN, immunonutrition; Arg, arginine; $\omega$-3-FAs, $\omega$-3-fatty acids; EN, enteral nutrition; PN, parenteral nutrition.

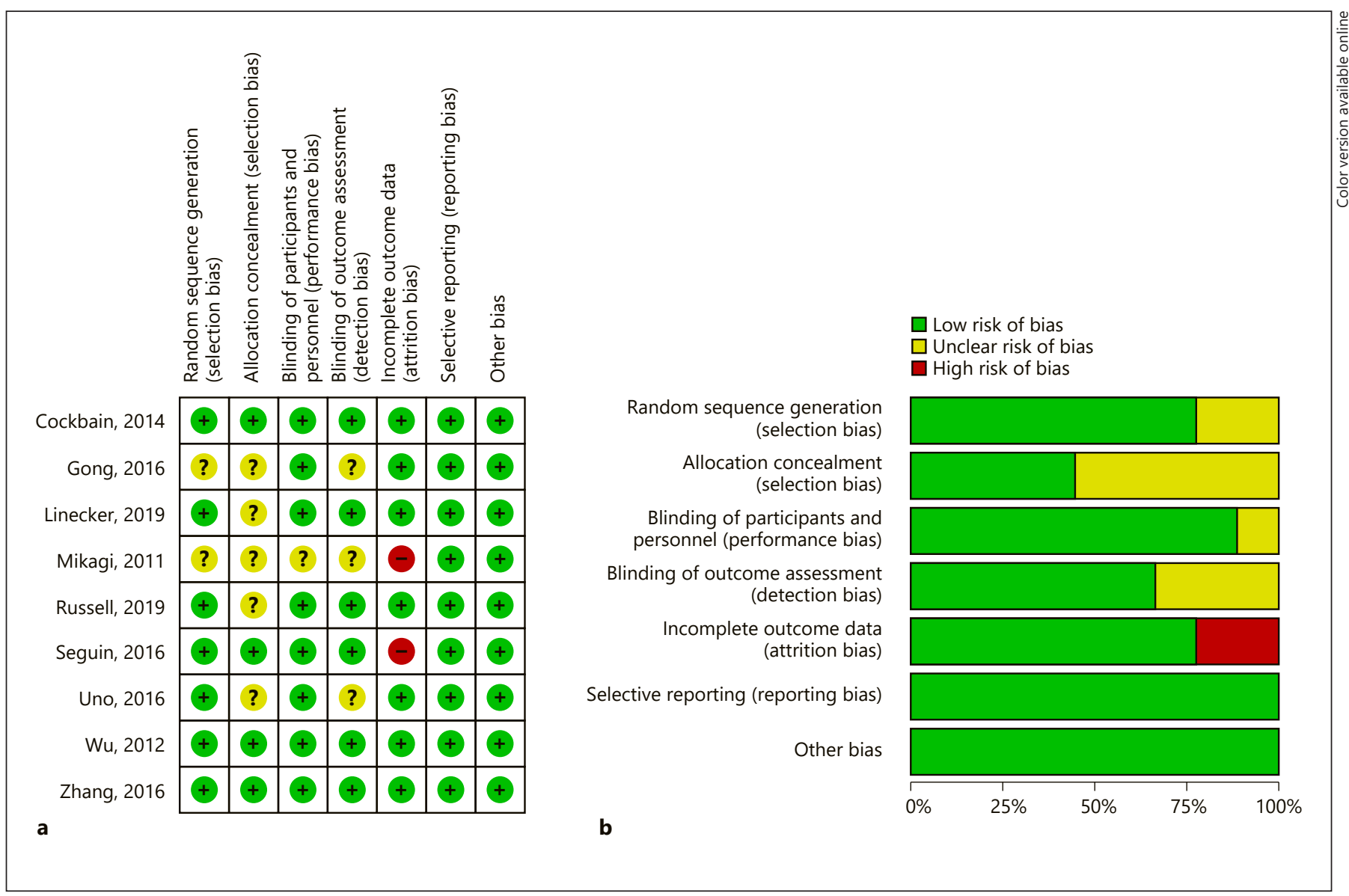

Fig. 2. Assessment of risk of bias for each eligible study $(n=9)$. a Risk of bias summary. b Risk of bias graph. 


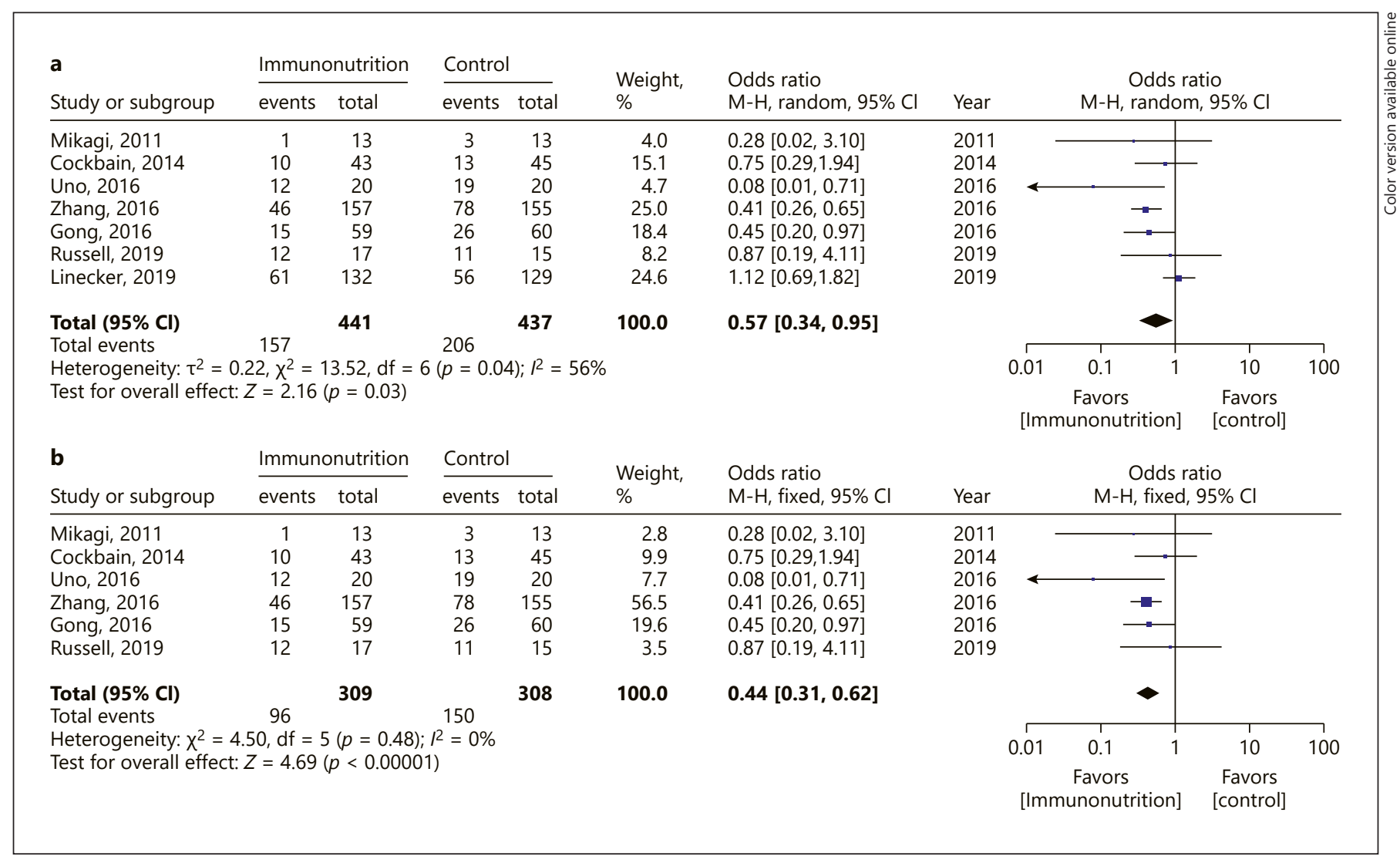

Fig. 3. a Forest plot on overall postoperative complications. b Forest plot on overall postoperative complications after removing Linecker's study.

$[23,25,28]$, and 2 trials were performed for comparing immunonutrition with regular diet $[21,29]$. Four studies $[21,25,26,29]$ were conducted with the oral immunonutrition diet IMPACT containing Arg, $\omega-3-F A s$, and RNA, with the remaining 5 studies using $\omega$-3-FAs only via the enteral or parenteral route. Immunonutrition was applied preoperatively in 6 studies $[21,23,25,26,28,29]$ and postoperatively in 3 studies. The duration of immunonutrition supplementation varied in these studies.

\section{Quality Assessment}

The bias risk of the included studies was assessed using the Cochrane Collaboration risk of bias tool. Seven articles $[22,23,25-29]$ reported the methods of random sequence generation, 4 studies $[22,23,25,27]$ performed allocation concealment, 8 studies [22-29] described the blinding of participants and personnel, and 6 studies [22, $23,25,27-29$ ] considered blinding of outcome assessment. Seven studies [22-24, 26-29] reported incomplete outcome data, while the remaining 2 trials $[21,25]$ were considered to have a high risk of attrition bias because of the high dropout rate. In all the studies, selective reporting and other bias were not identified. The bias assessment of the 9 eligible studies is shown in Figure 2.

\section{Effect of Immunonutrition on Overall Postoperative Complications}

Seven studies [21, 23, 24, 26-29] including 878 patients reported the overall postoperative complications. The meta-analysis showed that immunonutrition significantly reduced the incidence of overall postoperative complications ( $\mathrm{OR}=0.57,95 \% \mathrm{CI}: 0.34-0.95 ; p=0.03$ ). A random-effects model was used, as statistical heterogeneity was identified among the included studies $(p=0.04$, $I^{2}=56 \%$; Fig. 3a). Likewise, the pooled analysis remained unchanged and indicated that patients in the immunonutrition group had less overall postoperative complications $(\mathrm{OR}=0.44,95 \%$ CI: $0.31-0.62 ; p<0.00001)$ when the heterogeneity was reduced to zero $\left(p=0.48, I^{2}=0 \%\right)$, by excluding 1 trial conducted by Linecker et al. [28] (Fig. 3b). 


\begin{tabular}{|c|c|c|c|c|c|c|c|c|c|c|c|c|}
\hline \multirow{3}{*}{$\begin{array}{l}\text { a } \\
\text { Study or subgroup } \\
\text { Mikagi, } 2011\end{array}$} & \multicolumn{2}{|c|}{ Immunonutrition } & \multicolumn{2}{|l|}{ Control } & \multirow{3}{*}{$\begin{array}{l}\text { Weight, } \\
\% \\
\frac{1.6}{}\end{array}$} & \multirow{3}{*}{$\begin{array}{l}\text { Odds ratio } \\
\mathrm{M}-\mathrm{H}, \text { fixed, } 95 \% \mathrm{Cl} \\
0.31[0.01,8.30]\end{array}$} & \multirow{3}{*}{$\begin{array}{l}\text { Year } \\
2011\end{array}$} & \multirow{2}{*}{\multicolumn{4}{|c|}{$\begin{array}{c}\text { Odds ratio } \\
\mathrm{M}-\mathrm{H} \text {, fixed, } 95 \% \mathrm{Cl}\end{array}$}} & \\
\hline & \multirow{2}{*}{$\begin{array}{c}\text { events } \\
0\end{array}$} & \multirow{2}{*}{$\begin{array}{c}\text { total } \\
13\end{array}$} & \multicolumn{2}{|c|}{$\overline{\text { events total }}$} & & & & & & & & \\
\hline & & & 1 & $\frac{t a l}{13}$ & & & & 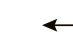 & & & & \\
\hline Wu, 2012 & 6 & 31 & 14 & 32 & 12.5 & $0.31[0.10,0.96]$ & 2012 & & & & & \\
\hline Cockbain, 2014 & 4 & 43 & 8 & 45 & 8.0 & $0.47[0.13,1.71]$ & 2014 & & & & & \\
\hline Zhang, 2016 & 15 & 157 & 30 & 155 & 30.7 & $0.44[0.23,0.86]$ & 2016 & & & & & \\
\hline Gong, 2016 & 8 & 59 & 16 & 60 & 15.4 & $0.43[0.17,1.10]$ & 2016 & & & & & \\
\hline Uno, 2016 & 8 & 20 & 15 & 20 & 10.1 & $0.22[0.06,0.86]$ & 2016 & & & & & \\
\hline Seguin, 2016 & 1 & 13 & 4 & 12 & 4.3 & $0.17[0.02,1.78]$ & 2016 & $\longleftarrow$ & & & & \\
\hline Russell, 2019 & 10 & 17 & 4 & 15 & 2.0 & $3.93[0.88,17.56]$ & 2019 & & & & & \\
\hline Linecker, 2019 & 14 & 132 & 15 & 129 & 15.3 & $0.90[0.42,1.95]$ & 2019 & & & & & \\
\hline Total $(95 \% \mathrm{Cl})$ & & 485 & & 481 & 100.0 & $0.53[0.37,0.75]$ & & & & & & \\
\hline Total events & 66 & & 107 & & & & & & & & & 1 \\
\hline Heterogeneity: $x^{2}=$ & $2.69, \mathrm{df}=$ & $8(p=0.12$ & $R^{2}=37 \%$ & & & & & 0.05 & 0.2 & 1 & 5 & 20 \\
\hline Test for overall effect & $Z=3.59$ & $(p=0.0003)$ & & & & & & {$[\mathrm{lmn}$} & $\begin{array}{l}\text { Favor } \\
\text { hunonu }\end{array}$ & & $\begin{array}{l}\text { Favors } \\
\text { [control] }\end{array}$ & \\
\hline b & Immunc & onutrition & Control & & & & & & & & & \\
\hline Study or subgroup & events & total & events & $\overline{\text { total }}$ & $\%$ & $\mathrm{M}-\mathrm{H}$, fixed, $95 \% \mathrm{Cl}$ & Year & & & 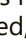 & $5 \% \mathrm{Cl}$ & \\
\hline Cockbain, 2014 & 1 & 43 & 4 & 45 & 11.7 & $0.24[0.03,2.28]$ & 2014 & & & & & \\
\hline Zhang, 2016 & 9 & 157 & 15 & 155 & 43.5 & $0.57[0.24,1.34]$ & 2016 & & & & & \\
\hline Gong, 2016 & 2 & 59 & 3 & 60 & 8.8 & $0.67[0.11,4.14]$ & 2016 & & & - & & \\
\hline Seguin, 2016 & 1 & 13 & 3 & 12 & 8.8 & $0.25[0.02,2.82]$ & 2016 & & & & & \\
\hline Uno, 2016 & 6 & 20 & 10 & 20 & 21.4 & $0.43[0.12,1.57]$ & 2016 & & & - & & \\
\hline Russell, 2019 & 2 & 17 & 2 & 15 & 5.7 & $0.87[0.11,7.05]$ & 2019 & & & & & \\
\hline Total $(95 \% \mathrm{Cl})$ & & 309 & & 307 & 100.0 & $0.50[0.28,0.89]$ & & & & & & \\
\hline Total events & 21 & & 37 & & & & & & & & & \\
\hline Heterogeneity: $\chi^{2}=$ & $21, d f=5$ & $(p=0.94)$ & $R=0 \%$ & & & & & 0.02 & 0.1 & 1 & 10 & 50 \\
\hline Test for overall effect & $Z=2.33$ & $(p=0.02)$ & & & & & & {$[\mathrm{lmm}$} & $\begin{array}{l}\text { Favors } \\
\text { unonut }\end{array}$ & & $\begin{array}{c}\text { Favors } \\
\text { [Control] }\end{array}$ & \\
\hline c & Immun & onutrition & Control & & Weight & Odds ratio & & & & & & \\
\hline Study or subgroup & events & total & events & $\overline{\text { total }}$ & $\%$ & $\mathrm{M}-\mathrm{H}$, fixed, $95 \% \mathrm{Cl}$ & Year & & & ed & $95 \% \mathrm{Cl}$ & \\
\hline Cockbain, 2014 & 0 & 43 & 4 & 45 & 16.6 & $0.11[0.01,2.03]$ & 2014 & & & & & \\
\hline Gong, 2016 & 3 & 59 & 6 & 60 & 21.6 & $0.48[0.11,2.03]$ & 2016 & & & - & & \\
\hline Zhang, 2016 & 6 & 157 & 6 & 155 & 22.2 & $0.99[0.31,3.13]$ & 2016 & & & & & \\
\hline Uno, 2016 & 1 & 20 & 3 & 20 & 10.9 & $0.30[0.03,3.15]$ & 2016 & & & & & \\
\hline Russell, 2019 & 3 & 17 & 3 & 15 & 10.0 & $0.86[0.15,5.06]$ & 2019 & & & & & \\
\hline Linecker, 2019 & 4 & 132 & 5 & 129 & 18.7 & $0.78[0.20,2.95]$ & 2019 & & & & & \\
\hline Total $(95 \% \mathrm{Cl})$ & & 428 & & 424 & 100.0 & $0.60[0.32,1.12]$ & & & & & & \\
\hline Total events & 17 & & 27 & & & & & & & & & \\
\hline Heterogeneity: $x^{2}=2$ & $75, \mathrm{df}=5$ & $(p=0.74)$ & $R=0 \%$ & & & & & 0.01 & 0.1 & 1 & 10 & 100 \\
\hline Test for overall effect & $Z=1.59$ & $(p=0.11)$ & & & & & & & $\begin{array}{r}\text { Favo } \\
\text { nunon }\end{array}$ & & $\begin{array}{l}\text { Favors } \\
\text { [control] }\end{array}$ & \\
\hline d & Immun & onutrition & Control & & Weight, & Odds ratio & & & & Is r & & \\
\hline Study or subgroup & events & total & events & $\overline{\text { total }}$ & $\%$ & $\mathrm{M}-\mathrm{H}$, fixed, $95 \% \mathrm{Cl}$ & Year & & & 1 & $95 \% \mathrm{Cl}$ & \\
\hline Mikagi, 2011 & 0 & 13 & 1 & 13 & 15.8 & $0.31[0.01,8.30]$ & 2011 & & & & - & \\
\hline Cockbain, 2014 & 3 & 43 & 0 & 45 & 4.9 & $7.86[0.39,156.90]$ & 2014 & & & & & $\rightarrow$ \\
\hline Seguin, 2016 & 0 & 13 & 2 & 12 & 27.3 & $0.16[0.01,3.60]$ & 2016 & $\leftarrow$ & & & & \\
\hline Gong, 2016 & 2 & 59 & 4 & 60 & 41.9 & $0.49[0.09,2.79]$ & 2016 & & & & & \\
\hline Russell, 2019 & 3 & 17 & 0 & 15 & 4.7 & $7.48[0.35,157.76]$ & 2019 & & & & & $\rightarrow$ \\
\hline Linecker, 2019 & 2 & 132 & 0 & 129 & 5.4 & $4.96[0.24,104.36]$ & 2019 & & & & & $\rightarrow$ \\
\hline Total $(95 \% \mathrm{Cl})$ & & 277 & & 274 & 100.0 & $1.30[0.55,3.08]$ & & & & & & \\
\hline Total events & 10 & & 7 & & & & & & & & 1 & $\square$ \\
\hline Heterogeneity: $x^{2}=7$ & $09, \mathrm{df}=5$ & $(p=0.21)$ & $R=30 \%$ & & & & & 0.01 & 0.1 & 1 & 10 & 100 \\
\hline Test for overall effect & $Z=0.60$ & $(p=0.55)$ & & & & & & {$[\mathrm{Im}$} & $\begin{array}{l}\text { Favol } \\
\text { nunoni }\end{array}$ & & $\begin{array}{l}\text { Favors } \\
\text { [control] }\end{array}$ & \\
\hline
\end{tabular}

Fig. 4. Forest plot on postoperative infectious complications. a Overall postoperative infectious complications. b Incision infection. c Pulmonary infection. d Urinary tract infection. 


\begin{tabular}{|c|c|c|c|c|c|c|c|c|c|c|c|c|}
\hline \multirow{3}{*}{$\frac{\text { Study or subgroup }}{\text { Uno, } 2016}$} & \multicolumn{2}{|c|}{ Immunonutrition } & \multicolumn{2}{|c|}{ Control } & \multirow{2}{*}{$\begin{array}{l}\text { Weight, } \\
\%\end{array}$} & \multirow{2}{*}{$\begin{array}{l}\text { Odds ratio } \\
\mathrm{M}-\mathrm{H} \text {, fixed, } 95 \% \mathrm{Cl}\end{array}$} & \multirow[b]{2}{*}{ Year } & \multirow{2}{*}{\multicolumn{5}{|c|}{$\begin{array}{c}\text { Odds ratio } \\
\mathrm{M}-\mathrm{H} \text {, fixed, } 95 \% \mathrm{Cl}\end{array}$}} \\
\hline & \multirow{2}{*}{$\frac{\text { events }}{2}$} & \multirow{2}{*}{$\begin{array}{r}\text { total } \\
20\end{array}$} & \multirow{2}{*}{$\frac{\text { events }}{5}$} & \multirow{2}{*}{$\begin{array}{r}\text { total } \\
20\end{array}$} & & & & & & & & \\
\hline & & & & & 29.3 & $0.33[0.06,1.97]$ & 2016 & & 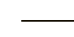 & - & & \\
\hline Gong, 2016 & 1 & 59 & 2 & 60 & 12.7 & $0.50[0.04,5.67]$ & 2016 & & & & & \\
\hline Zhang, 2016 & 2 & 157 & 7 & 155 & 45.3 & $0.27[0.06,1.33]$ & 2016 & & 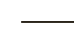 & - & & \\
\hline Linecker, 2019 & 4 & 132 & 2 & 129 & 12.8 & $1.98[0.36,11.03]$ & 2019 & & & & & \\
\hline Total $(95 \% \mathrm{Cl})$ & & 368 & & 364 & 100.0 & $0.54[0.23,1.24]$ & & & & & & \\
\hline $\begin{array}{l}\text { Total events } \\
\text { Heterogeneity: } \chi^{2}=\end{array}$ & $21, d f=3$ & $(p=0.36)$ & $\begin{array}{l}16 \\
2=7 \%\end{array}$ & & & & & 0.01 & 0.1 & 1 & 10 & $\sqrt{100}$ \\
\hline Test for overall effec & $z=1.45$ & $p=0.15)$ & & & & & & {$[\mathrm{Imn}$} & $\begin{array}{l}\text { Favor } \\
\text { unonu }\end{array}$ & & $\begin{array}{l}\text { Favors } \\
\text { [control] }\end{array}$ & \\
\hline
\end{tabular}

Fig. 5. Forest plot on liver failure.

\begin{tabular}{|c|c|c|c|c|c|c|c|c|c|c|c|c|}
\hline \multirow{3}{*}{$\frac{\text { Study or subgroup }}{\mathrm{Wu}, 2012}$} & \multicolumn{2}{|c|}{ Immunonutrition } & \multicolumn{2}{|l|}{ Control } & \multirow{2}{*}{$\begin{array}{l}\text { Weight, } \\
\%\end{array}$} & \multirow{2}{*}{$\begin{array}{l}\text { Odds ratio } \\
\mathrm{M}-\mathrm{H} \text {, fixed, } 95 \% \mathrm{Cl}\end{array}$} & \multirow[b]{2}{*}{ Year } & \multirow{2}{*}{\multicolumn{5}{|c|}{$\begin{array}{c}\text { Odds ratio } \\
\mathrm{M}-\mathrm{H} \text {, fixed, } 95 \% \mathrm{Cl}\end{array}$}} \\
\hline & \multirow{2}{*}{$\frac{\text { events }}{0}$} & \multirow{2}{*}{$\begin{array}{r}\text { total } \\
31\end{array}$} & \multirow{2}{*}{$\begin{array}{c}\text { events } \\
0\end{array}$} & \multirow{2}{*}{$\begin{array}{r}\text { total } \\
32\end{array}$} & & & & & & & & \\
\hline & & & & & & Not estimable & 2012 & & & & & \\
\hline Zhang, 2016 & 1 & 157 & 5 & 155 & 50.7 & $0.19[0.02,1.67]$ & 2016 & & & & & \\
\hline Uno, 2016 & 0 & 20 & 0 & 20 & & Not estimable & 2016 & & & & & \\
\hline Gong, 2016 & 0 & 59 & 1 & 60 & 14.9 & $0.33[0.01,8.35]$ & 2016 & & & & & \\
\hline Linecker, 2019 & 5 & 132 & 2 & 129 & 19.7 & $2.50[0.48,13.12]$ & 2019 & & & & & \\
\hline $\begin{array}{l}\text { Total events } \\
\text { Heterogeneity: } \chi^{2}=\end{array}$ & $04, d f=3$ & $3(p=0.26)$ & $\begin{array}{c}9 \\
R=26 \%\end{array}$ & & & & & 0.01 & 0.1 & 1 & 10 & $\sqrt{100}$ \\
\hline Test for overall effe & $Z=0.74$ & $(p=0.46)$ & & & & & & {$[\operatorname{lm}$} & $\begin{array}{l}\text { Favo } \\
\text { tunont }\end{array}$ & & $\begin{array}{l}\text { Favors } \\
\text { [control] }\end{array}$ & \\
\hline
\end{tabular}

Fig. 6. Forest plot on postoperative mortality.

Effect of Immunonutrition on Postoperative Infectious Complications

Nine studies [21-29] including 966 patients reported overall postoperative infectious complications, 6 studies $[23-27,29]$ including 616 participants explored incision infection, 6 studies [23, 24, 26-29] enrolling 852 patients investigated pulmonary infection, and 6 studies $[21,23-$ $25,28,29]$ recruiting 551 patients documented urinary tract infection. Pooled analysis showed that the incidence of overall postoperative infectious complications was significantly reduced in the immunonutrition group than in the control group $(\mathrm{OR}=0.53,95 \%$ CI: $0.37-0.75 ; p=$ $0.0003)$. A fixed-effects model was used because there was no statistical heterogeneity ( $p=0.12, I^{2}=37 \%$; Fig. $4 a$ ). Besides, fewer incision infections occurred in the immunonutrition group $(\mathrm{OR}=0.50,95 \% \mathrm{CI}: 0.28-0.89 ; p=$ 0.02 ; Fig. $4 \mathrm{~b}$ ), while no significant differences were found in pulmonary infection $(\mathrm{OR}=0.60,95 \% \mathrm{CI}$ : $0.32-1.12$; $p=0.11$; Fig. $4 \mathrm{c})$ and urinary tract infection $(\mathrm{OR}=1.30$, $95 \%$ CI: $0.55-3.08$; $p=0.55$; Fig. $4 \mathrm{~d}$ ) between the 2 groups.

\section{Effect of Immunonutrition on Liver Failure}

Four studies [24, 26-28] included 732 patients and analyzed the effect of immunonutrition supplementation on liver failure. The meta-analysis indicated that there was no significant difference in liver failure between the 2 groups (OR $=0.54,95 \% \mathrm{CI}: 0.23-1.24 ; p=0.15)$. A fixed-effects model was used as there was no heterogeneity among the studies ( $p=0.36, I^{2}=7 \%$; Fig. 5 ).

\section{Effect of Immunonutrition on Postoperative Mortality}

Six studies [22-24, 26-28] including 883 participants reported the index regarding postoperative mortality. The meta-analysis indicated that there was no significant difference in postoperative mortality between the immunonutrition group and the control group $(\mathrm{OR}=0.69,95 \%$ 


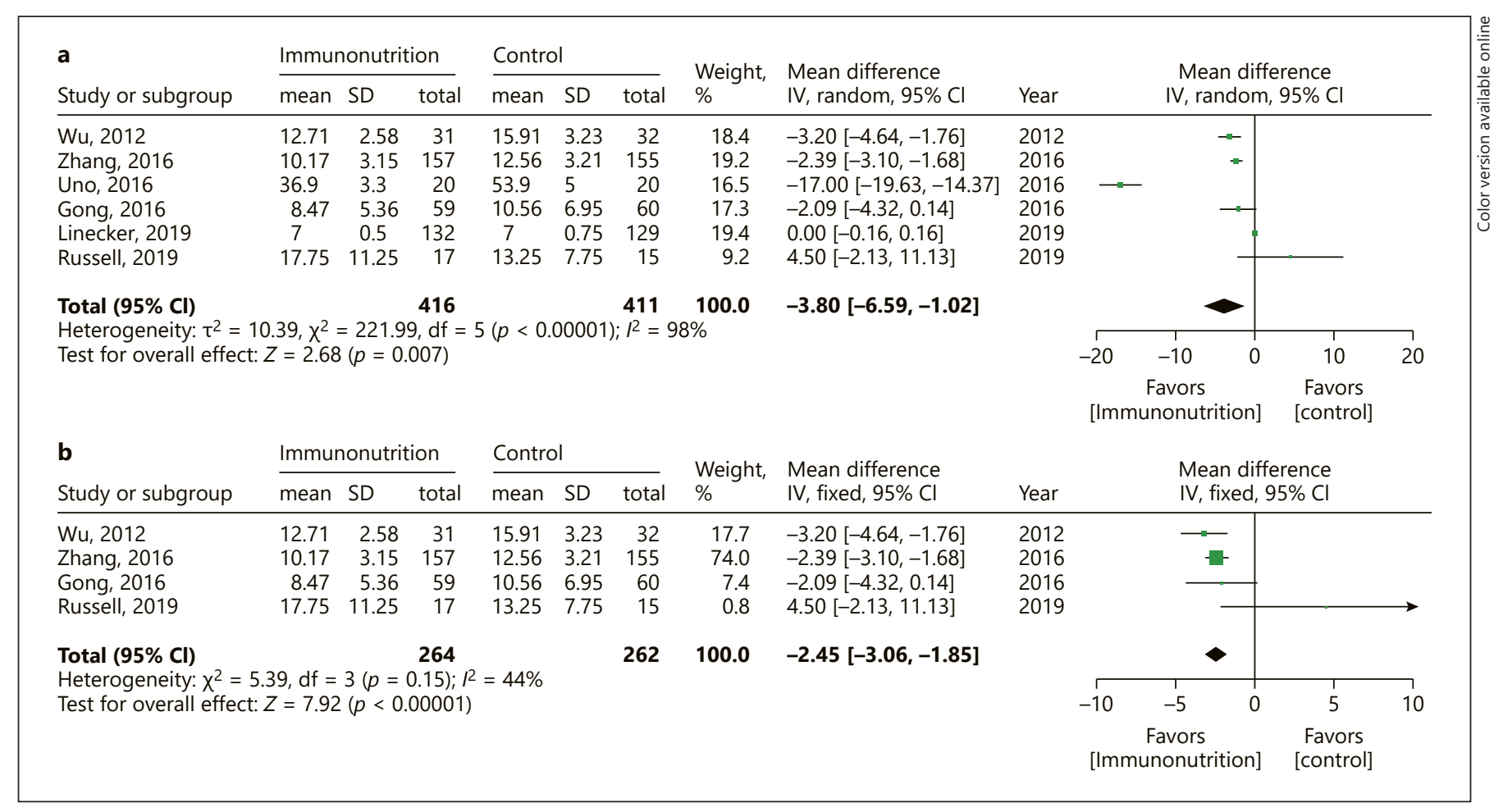

Fig. 7. a Forest plot on length of hospital stay. b Forest plot on length of hospital stay after removing 2 studies.

CI: $0.26-1.83 ; p=0.46)$. There was no significant heterogeneity among these studies; thus, a fixed-effects model was adopted ( $p=0.26, I^{2}=26 \%$; Fig. 6).

\section{Effect of Immunonutrition on Length of Hospital Stay}

Six studies [22, 24, 26-29] enrolling 827 patients provided data concerning length of hospital stay. Pooled analysis showed that there was a significantly shorter length of hospital stay in the immunonutrition group compared with the control group ( $\mathrm{MD}=-3.80,95 \% \mathrm{CI}$ : -6.59 to $-1.02 ; p=0.007)$. A random-effects model was used, due to statistical heterogeneity $\left(p<0.00001, I^{2}=\right.$ $98 \%$; Fig. 7a). To reduce the heterogeneity, 2 studies [ 26 , 28 ] were removed ( $\left.p=0.15, I^{2}=44 \%\right)$. The recalculated results also showed that immunonutrition could significantly shorten the length of hospital stay $(\mathrm{MD}=-2.45$, $95 \%$ CI: -3.06 to -1.85 ; $p<0.00001$; Fig. $7 b)$.

\section{Subgroup Analysis}

Subgroup analyses were further conducted on the basis of the timing of initiation and route of immunonutrition supplementation, including preoperative enteral nutrition (EN), preoperative parenteral nutrition (PN), and postoperative PN. The results indicated that postoperative $\mathrm{PN}$ was associated with significant decreases in overall postoperative complications, infectious complications, and length of hospital stay. The results are shown in Table 2.

\section{Discussion}

Hepatectomy is an intervention with a considerable incidence of postoperative complications and mortality. Most of the patients undergoing liver resection are often have varying degrees of hepatitis or cirrhosis and malnutrition that further exacerbate the adverse outcomes after surgery [32]. There is increasing evidence that perioperative nutrition can improve the prognosis after major surgery; therefore, it is recommended as an indispensable adjunctive care in these patients [18, 33, 34].

Immunonutrition, including $\omega-3$-FAs, Gln, Arg, and nucleotides, has been receiving increasing attention for the purpose of improving the nutritional status and immune response of patients who have undergone hepatectomy. $\omega$-3-FAs exert anti-inflammatory effects by modulating 
the synthesis of different eicosanoids and competing with arachidonic acid for cyclo-oxygenase metabolism at the cell membrane $[35,36]$. Gln can not only be used as an oxidative fuel for rapidly replicating cells, but it also has a protective or restorative effect on the gastrointestinal tract [37]. Arg, a semiessential amino acid, has positive effects on reparative collagen synthesis and immune function, and it is considered to be a fortifier of T-cells [38]. Nucleotides are essential components of RNA, which can improve hypoimmunity and enhance host defenses in patients with cancer $[36,39]$. Recently, researchers have demonstrated that immunonutrition has the potential to improve the host immune state, reduce infectious complications, and shorten the length of hospital stay after major abdominal surgeries, such as gastrointestinal surgery [13-16], pancreaticoduodenectomy [40], and liver transplantation [41]. Despite the reported benefits of this treatment, we are only aware of 1 published meta-analysis [42] exploring the effects of immunonutrition on clinical outcomes in patients who undergo liver resection. Controversy still persists regarding whether or not perioperative immunonutrition could offer substantial benefits to patients undergoing hepatectomy as compared with standard nutrition.

This systematic review and meta-analysis demonstrated that perioperative immunonutrition supplementation, compared with the control group, could significantly improve overall postoperative complications, postoperative infectious complications, and incision infection in patients undergoing liver resection. It is well known that the supply of immunomodulatory nutrients can promote the postoperative maintenance of homeostasis, modulate the inflammatory response, and improve the host immune status [12]. Similar to these reported results, our pooled results further corroborated its clinical efficacy in patients undergoing liver resection. Furthermore, the length of hospital stay was significantly shorter in the immunonutrition group. Studies have reported that frequent complications and malnutrition were associated with longer hospital stay [43]. In our study, decreased postoperative complications and improvement of nutritional status may have led to shortening of the length of hospitalization. In addition, the meta-analysis showed that the incidence of other infectious complications, such as pneumonia and urinary tract infection, in the immunonutrition group were comparable to those in the control group. With respect to liver failure and mortality, our analysis also failed to show any statistical difference between the 2 groups. These results confirm the safety of immunonutrition and are in accordance with the results reported by the majority of the included studies.

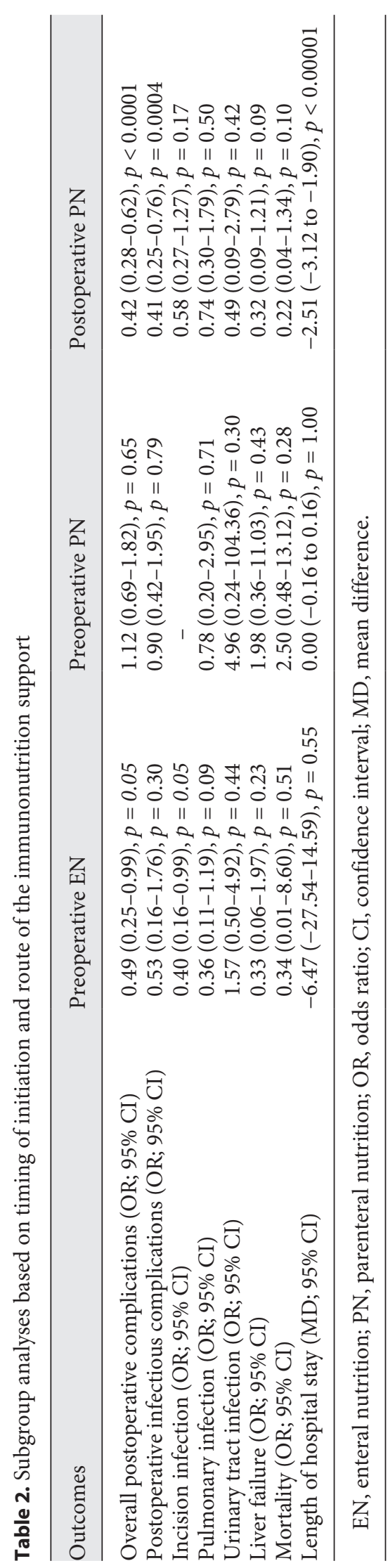

Ann Nutr Metab 2020;76:375-386 DOI: $10.1159 / 000509979$
Perioperative Immunonutrition and

Hepatectomy, a Meta-Analysis of RCTs 
Since different schemes of immunonutrition administration were designed across studies, we conducted subgroup analyses through changing the inclusion criteria to further evaluate the effects of immunonutrition. The results showed that relevant improvements in most clinical indicators could be noted in both the preoperative EN and postoperative PN groups, but significant beneficial effects of immunonutrition on overall postoperative complications, infectious complications, and length of hospital stay were observed only in the postoperative PN group. These results are roughly consistent with the conclusions of previous studies [44, 45], and they provide a new basis for the use of postoperative immunonutrition in patients undergoing hepatectomy. Furthermore, because considerable heterogeneity was found among the included studies in terms of 2 outcomes (overall postoperative complications and length of hospital stay), the random-effects model was used in our analysis. Sensitivity analysis was performed by removing one study at a time and repeating the meta-analysis to assess whether any one study significantly affected the pooled estimates. However, sensitivity analyses and application of different statistical methods did not reveal any significant changes in the outcome estimates, thereby strengthening the results of this meta-analysis.

Similar results have been reported in some previous studies. A meta-analysis conducted by Zhang et al. [42] included 8 RCTs involving a total of 805 patients, of which 402 patients received immunonutrition and 403 did not receive immunonutrition, and they assessed the effects of immunonutrition on clinical outcomes in patients undergoing hepatectomy. Their study showed that immunonutrition could effectively reduce the incidence of overall and infectious postoperative complications and shorten the hospital stay. Besides, their subgroup analysis revealed that $\omega-3$-FA supplementation was also associated with a significantly lower risk of postoperative total complications and infectious complications and shorter length of hospital stay. Meanwhile, the postoperative mortality was not affected. In addition, another metaanalysis [20] explored the influence of $\omega-3-F A s$ on both liver function and inflammatory reaction in patients undergoing liver resection. Compared with the control group, several liver function markers (such as alanine aminotransferase, aspartate aminotransferase, and prealbumin) and inflammatory reaction markers (such as white blood cell count and C-reactive protein) in the $\omega$-3FA group were significantly improved. These results indicated that $\omega$-3-FA administration had a positive impact on the liver function and inflammatory reaction after surgery.

The major strength of this systematic review and metaanalysis, compared with the study conducted by Zhang et al. [42], was a comprehensive analysis of the effects of immunonutrition on clinical indicators in patients undergoing hepatectomy. In addition, a rigorous screening process was independently conducted by 3 researchers, thus resulting in more accurate estimates of effects. Moreover, we performed subgroup analyses based on the timing of initiation and route of immunonutrition to further assess the effects. Even so, there are several limitations that need to be described. First, this review included only 9 RCTs, which might influence the credibility of the results. Second, we only included studies published in English language, and therefore, some relevant articles in other languages might have been missed. Third, the variety of baseline characteristics and the different schemes of immunonutrition supplementation might have led to heterogeneity. Although subgroup analyses were conducted to overcome this limitation, its influence might not have been completely controlled. Finally, laboratory indices were not pooled for analysis due to the incomplete data.

\section{Conclusions}

This systematic review and meta-analysis showed that perioperative administration of immunonutrition containing $\omega-3-F A s$ can improve the overall postoperative complications, postoperative infectious complications, and incision infection, and it can shorten the length of hospital stay. Therefore, immunonutrition is clinically safe and feasible to be recommended as nutritional support for patients undergoing hepatectomy. However, considering the small number of patients included in this meta-analysis, more high-quality, large-sample, and multicenter RCTs are still required to verify the reliability of our conclusion.

\section{Statement of Ethics}

This review was performed in accordance with the guidelines of the Preferred Reporting Items for Systematic Reviews and MetaAnalyses (PRISMA) Statement. The prospective protocol for this study has been registered in the International Prospective Register of Systematic Review (PROSPERO), and the registration number is CRD42020163591. 


\section{Conflict of Interest Statement}

The authors declare that they have no conflicts of interest.

\section{Funding Sources}

This work was supported by the Southwest Medical University Foundation (2018-ZRZD-010) and Doctoral Startup Fund of Affiliated Hospital of Southwest Medical University.

\section{Author Contributions}

B.G. contributed to the study conception and design, data extraction, and the drafting of the manuscript; J.L. (equal contributor) contributed to data extraction, quality assessments, analysis of data, and the drafting of the manuscript. Y.L. contributed to data extraction, quality assessments, and analysis of data. F.Z. contributed to quality assessments and analysis of data. X.Y., Y.G., and S.S. contributed to the analysis and interpretation of the descriptive data. B.L. (corresponding author) contributed to study conception and design and the critical revision. All authors have read and approved the final manuscript.

\section{References}

1 Poon RT, Fan ST, Lo CM, Liu CL, Lam CM, Yuen WK, et al. Improving perioperative outcome expands the role of hepatectomy in management of benign and malignant hepatobiliary diseases: analysis of 1222 consecutive patients from a prospective database. Ann Surg. 2004 Oct;240(4):698-10;

2 Cescon M, Vetrone G, Grazi GL, Ramacciato G, Ercolani G, Ravaioli M, et al. Trends in perioperative outcome after hepatic resection: analysis of 1500 consecutive unselected cases over 20 years. Ann Surg. 2009 Jun; 249(6):995-1002.

3 Dokmak S, Ftériche FS, Borscheid R, Cauchy F, Farges O, Belghiti J. 2012 Liver resections in the 21st century: we are far from zero mortality. HPB. 2013 Nov;15(11):908-15.

4 Kingham TP, Correa-Gallego C, D'Angelica MI, Gönen M, DeMatteo RP, Fong Y, et al. Hepatic parenchymal preservation surgery: decreasing morbidity and mortality rates in 4,152 resections for malignancy. J Am Coll Surg. 2015 Apr;220(4):471-9.

5 Kamiyama T, Nakanishi K, Yokoo H, Kamachi H, Tahara M, Yamashita K, et al. Perioperative management of hepatic resection toward zero mortality and morbidity: analysis of 793 consecutive cases in a single institution. J Am Coll Surg. 2010 Oct;211(4):443-9.

6 She WH, Chan AC, Cheung TT, Chok KS, Dai WC, Chan SC, et al. Short- and long-term impact of reoperation for complications after major hepatectomy for hepatocellular carcinoma. Surgery. 2016 Nov; 160(5):1236-43

7 Cheung K, Lee SS, Raman M. Prevalence and mechanisms of malnutrition in patients with advanced liver disease, and nutrition management strategies. Clin Gastroenterol Hepatol. $2012 \mathrm{Feb} ; 10(2): 117-25$.

8 Merli M, Nicolini G, Angeloni S, Riggio O. Malnutrition is a risk factor in cirrhotic patients undergoing surgery. Nutrition. 2002 Nov-Dec;18(11-12):978-86.

9 Merli M, Giusto M, Gentili F, Novelli G, Ferretti G, Riggio O, et al. Nutritional status: its influence on the outcome of patients undergoing liver transplantation. Liver Int. 2010 Feb;30(2):208-14.
10 Masuda T, Shirabe K, Yoshiya S, Matono R, Morita K, Hashimoto N, et al. Nutrition support and infections associated with hepatic resection and liver transplantation in patients with chronic liver disease. JPEN J Parenter Enteral Nutr. 2013 May-Jun;37(3):318-26.

11 Braga M. Perioperative immunonutrition and gut function. Curr Opin Clin Nutr Metab Care. 2012 Sep;15(5):485-8.

12 Calder PC. Immunonutrition in surgical and critically ill patients. Br J Nutr. 2007 Oct: 98(Suppl 1):S133-9.

13 Cheng Y, Zhang J, Zhang L, Wu J, Zhan Z. Enteral immunonutrition versus enteral nutrition for gastric cancer patients undergoing a total gastrectomy: a systematic review and meta-analysis. BMC Gastroenterol. 2018 Jan; 18(1):11.

14 Luo Z, Wang J, Zhang Z, Li H, Huang L, Qiao $Y$, et al. Efficacy of early enteral immunonutrition on immune function and clinical outcome for postoperative patients with gastrointestinal cancer. JPEN J Parenter Enteral Nutr. 2018 May;42(4):758-65.

15 Adiamah A, Skořepa $\mathrm{P}$, Weimann A, Lobo DN. The impact of preoperative immune modulating nutrition on outcomes in patients undergoing surgery for gastrointestinal cancer: a systematic review and meta-analysis. Ann Surg. 2019 Aug;270(2):247-56.

16 Wong CS, Aly EH. The effects of enteral immunonutrition in upper gastrointestinal surgery: a systematic review and meta-analysis. Int J Surg. 2016 May;29:137-50.

17 Braga M, Gianotti L, Nespoli L, Radaelli G, Di Carlo V. Nutritional approach in malnourished surgical patients: a prospective randomized study. Arch Surg. 2002 Feb;137(2):17480.

18 Weimann A, Braga M, Carli F, Higashiguchi T, Hübner M, Klek S, et al. ESPEN guideline: clinical nutrition in surgery. Clin Nutr. 2017 Jun;36(3):623-50.

19 Arends J, Bachmann P, Baracos V, Barthelemy N, Bertz H, Bozzetti F, et al. ESPEN guidelines on nutrition in cancer patients. Clin Nutr. 2017 Feb;36(1):11-48.
20 Zhang C, Lin J, Li F, Li Y, Jiang B, Bai H, et al. Effect of $\omega-3$ polyunsaturated fatty acids on liver function and inflammatory reaction in patients undergoing hepatectomy: a systematic review and meta-analysis of randomized control trials. Expert Rev Gastroenterol Hepatol. 2019 Apr;13(4):375-84.

21 Mikagi K, Kawahara R, Kinoshita H, Aoyagi S. Effect of preoperative immunonutrition in patients undergoing hepatectomy; a randomized controlled trial. Kurume Med J. 2011; 58(1):1-8.

22 Wu Z, Qin J, Pu L. Omega-3 fatty acid improves the clinical outcome of hepatectomized patients with hepatitis B virus (HBV)associated hepatocellular carcinoma. J Biomed Res. 2012 Nov;26(6):395-9.

23 Cockbain AJ, Volpato M, Race AD, Munarini A, Fazio C, Belluzzi A, et al. Anticolorectal cancer activity of the omega-3 polyunsaturated fatty acid eicosapentaenoic acid. Gut. 2014 Nov;63(11):1760-8.

24 Gong Y, Liu Z, Liao Y, Mai C, Chen T, Tang $\mathrm{H}$, et al. Effectiveness of $\omega-3$ polyunsaturated fatty acids based lipid emulsions for treatment of patients after hepatectomy: a prospective clinical trial. Nutrients. 2016 Jun;8(6):357.

25 Seguin P, Locher C, Boudjema K, Hamon C, Mouchel C, Malledant Y, et al. Effect of a perioperative nutritional supplementation with Oral Impact ${ }^{\oplus}$ in patients undergoing hepatic surgery for liver cancer: a prospective, placebo-controlled, randomized, doubleblind study. Nutr Cancer. 2016 Mar;68(3): 464-72.

26 Uno H, Furukawa K, Suzuki D, Shimizu H, Ohtsuka M, Kato A, et al. Immunonutrition suppresses acute inflammatory responses through modulation of resolvin $\mathrm{E} 1$ in patients undergoing major hepatobiliary resection. Surgery. 2016 Jul;160(1):228-36.

27 Zhang B, Wei G, Li R, Wang Y, Yu J, Wang R, et al. $\mathrm{n}-3$ fatty acid-based parenteral nutrition improves postoperative recovery for cirrhotic patients with liver cancer: a randomized controlled clinical trial. Clin Nutr. 2017 Oct; 36(5):1239-44 
28 Linecker M, Botea F, Aristotele Raptis D, Nicolaescu D, Limani P, Alikhanov R, et al. Perioperative omega-3 fatty acids fail to confer protection in liver surgery: results of a multicentric, double-blind, randomized controlled trial. J Hepatol. 2020 Mar;72(3):498505.

29 Russell K, Zhang HG, Gillanders LK, Bartlett AS, Fisk HL, Calder PC, et al. Preoperative immunonutrition in patients undergoing liver resection: a prospective randomized trial. World J Hepatol. 2019 Mar;11(3):305-17.

30 Moher D, Shamseer L, Clarke M, Ghersi D, Liberati A, Petticrew M, et al. Preferred reporting items for systematic review and metaanalysis protocols (PRISMA-P) 2015 statement. Syst Rev. 2015 Jan;4:1.

31 Higgins JP, Altman DG, Gøtzsche PC, Jüni P, Moher D, Oxman AD, et al. The Cochrane Collaboration's tool for assessing risk of bias in randomised trials. BMJ. 2011 Oct;343: d5928.

32 Maharshi S, Sharma BC, Srivastava S. Malnutrition in cirrhosis increases morbidity and mortality. J Gastroenterol Hepatol. 2015 Oct; 30(10):1507-13.

33 Stratton RJ, Elia M. Who benefits from nutritional support: what is the evidence? Eur J Gastroenterol Hepatol. 2007 May;19(5):3538.
34 Koretz RL, Avenell A, Lipman TO. Nutritional support for liver disease. Cochrane Database Syst Rev. 2012 May;(5):CD008344.

35 Alexander JW. Immunonutrition: the role of omega-3 fatty acids. Nutrition. 1998 Jul-Aug; 14(7-8):627-33.

36 Schloerb PR. Immune-enhancing diets: products, components, and their rationales. JPEN J Parenter Enteral Nutr. 2001 Mar-Apr;25(2 Suppl):S3-7.

37 Curi R, Lagranha CJ, Doi SQ, Sellitti DF, Procopio J, Pithon-Curi TC, et al. Molecular mechanisms of glutamine action. J Cell Physiol. 2005 Aug;204(2):392-401.

38 Reynolds JV, Daly JM, Zhang S, Evantash E, Shou J, Sigal R, et al. Immunomodulatory mechanisms of arginine. Surgery. 1988 Aug; 104(2):142-51.

39 Hess JR, Greenberg NA. The role of nucleotides in the immune and gastrointestinal systems: potential clinical applications. Nutr Clin Pract. 2012 Apr;27(2):281-94.

40 Guan H, Chen S, Huang Q. Effects of enteral immunonutrition in patients undergoing pancreaticoduodenectomy: a meta-analysis of randomized controlled trials. Ann Nutr Metab. 2019;74(1):53-61.
41 Lei Q, Wang X, Zheng H, Bi J, Tan S, Li N. Peri-operative immunonutrition in patients undergoing liver transplantation: a metaanalysis of randomized controlled trials. Asia Pac J Clin Nutr. 2015;24(4):583-90.

42 Zhang C, Chen B, Jiao A, Li F, Wang B, Sun $\mathrm{N}$, et al. The benefit of immunonutrition in patients undergoing hepatectomy: a systematic review and meta-analysis. Oncotarget. 2017 Aug;8(49):86843-52.

43 Huang TH, Hsieh CC, Kuo LM, Chang CC, Chen $\mathrm{CH}$, Chi CC, et al. Malnutrition associated with an increased risk of postoperative complications following hepatectomy in patients with hepatocellular carcinoma. HPB. 2019 Sep;21(9):1150-5.

44 Marimuthu K, Varadhan KK, Ljungqvist O, Lobo DN. A meta-analysis of the effect of combinations of immune modulating nutrients on outcome in patients undergoing major open gastrointestinal surgery. Ann Surg. 2012 Jun;255(6):1060-8.

45 Osland E, Hossain MB, Khan S, Memon MA. Effect of timing of pharmaconutrition (immunonutrition) administration on outcomes of elective surgery for gastrointestinal malignancies: a systematic review and meta-analysis. JPEN J Parenter Enteral Nutr. 2014 Jan; 38(1):53-69. 